\title{
L'EXPÉRIENCE ZAPATISTE ET LA QUESTION INDIGÈNE AU MEXIQUE
}

\author{
Arnaud Martin
}

Le Seuil | « Pouvoirs »

2019/4 N $171 \mid$ pages 85 à 96

ISSN 0152-0768

ISBN 9782021432497

Article disponible en ligne à l'adresse :

https://www.cairn.info/revue-pouvoirs-2019-4-page-85.htm

Distribution électronique Cairn.info pour Le Seuil.

(C) Le Seuil. Tous droits réservés pour tous pays.

La reproduction ou représentation de cet article, notamment par photocopie, n'est autorisée que dans les limites des conditions générales d'utilisation du site ou, le cas échéant, des conditions générales de la licence souscrite par votre établissement. Toute autre reproduction ou représentation, en tout ou partie, sous quelque forme et de quelque manière que ce soit, est interdite sauf accord préalable et écrit de l'éditeur, en dehors des cas prévus par la législation en vigueur en France. Il est précisé que son stockage dans une base de données est également interdit. 


\section{Arnaud Martin}

\section{L'EXPÉRIENCE ZAPATISTE \\ ET LA QUESTION INDIGĖNE \\ AU MEXIQUE}

e $1^{\text {er }}$ janvier 1994 devait être un jour historique pour le Mexique, avec

l'entrée en vigueur de l'Accord de libre-échange nord-américain, conclu avec les États-Unis et le Canada (ALENA). Il le fut, mais par un événement aussi invraisemblable qu'inattendu: l'attaque des cinq plus grandes villes de l'État du Chiapas, l'État le plus pauvre, le moins peuplé et le plus reculé de la Fédération, à l'extrémité sud du pays, par des rebelles mal équipés et mal préparés composant l'Armée zapatiste de libération nationale (EZLN), une organisation révolutionnaire créée le 17 novembre 1983 par d'anciens membres de mouvements issus de la révolte universitaire de 1968. Ainsi faisait irruption, sur la scène politique mexicaine, une gauche radicale qui se proclamait «zapatiste», se considérant comme héritière du révolutionnaire mexicain Emiliano Zapata. Des centaines d'indigènes descendirent des montagnes, occupèrent les édifices publics, ouvrirent les portes des prisons, affichèrent partout la première déclaration de la forêt Lacandon, avant de se replier dans les forêts des hautes terres et d'opposer à l'armée mexicaine une résistance qui conduisit l'État à décréter un cessezle-feu unilatéral après douze jours de combat et à ouvrir des négociations le 17 janvier. Entre-temps, le président Carlos Salinas avait limogé le ministre de l'Intérieur et nommé son plus proche collaborateur, Manuel Camacho, à la tête d'une Commission pour la paix et la réconciliation au Chiapas.

En quelques jours, les néozapatistes ${ }^{1}$ étaient parvenus à leurs fins: forcer le pouvoir à ouvrir des négociations en vue d'obtenir une amélioration

1. Nous préférons parler de «néozapatisme» pour éviter toute confusion avec le «zapatisme» historique de la révolution mexicaine. 
des conditions de vie des indigènes chiapanèques, lesquels représentent $22 \%$ des habitants du Chiapas, mais moins de $1 \%$ de la population mexicaine, et sont de très loin les Mexicains les plus pauvres du plus pauvre État du Mexique 2 .

Cependant, le néozapatisme ne se résume ni à un soulèvement armé éphémère, ni à une idéologie ou à une doctrine, ni même à la défense de la cause indigène, à laquelle on l'assimile souvent. On doit davantage le définir comme une expérience de transformation sociale qui a pris la forme d'une gestion autonome de certaines zones du Chiapas par le biais de structures administratives parallèles à celles de l'État. À partir de la défense de revendications indigènes, il a démontré qu'il était possible de mettre en place une politique alternative au néolibéralisme, rendu responsable de la misère des peuples indigènes. À l'origine mouvement révolutionnaire qui a épousé le combat pour le respect des droits des peuples indigènes du Chiapas, le néozapatisme est ainsi devenu un modèle altermondialiste, revenant à ses origines anticapitalistes et à l'inspiration sociale des premiers défenseurs de la cause indigène.

\section{UNE PRISE EN COMPTE AMBIGUË DE LA CAUSE INDIGÈNE}

Le néozapatisme a toujours entretenu le doute sur sa véritable nature. Organisation révolutionnaire ou mouvement indigène, l'ambiguïté n'a jamais été levée, voire a été habilement entretenue ${ }^{3}$. Semblant hésiter entre la rébellion contre le pouvoir central et la défense des droits indigènes, sans que la première soit forcément motivée par la seconde, le néozapatisme ne s'est jamais départi d'un caractère révolutionnaire en contradiction avec les aspirations des populations indigènes, même si son projet principal était moins la conquête du pouvoir que la mise en œuvre d'un projet altermondialiste.

\section{Le néozapatisme est un mouvement révolutionnaire}

$\mathrm{Au}$ regard de ses origines et de ses premières manifestations, le néozapatisme présente certains traits d'une organisation révolutionnaire. L'EZLN est issue d'une autre formation, les Forces de libération nationale, lesquelles, créées en 1969 par des jeunes de Monterrey et intégrant les membres

2. $70 \%$ des Chiapanèques vivent en dessous du seuil de pauvreté, et $20 \%$ des indigènes du Chiapas n'ont aucun revenu monétaire.

3. Cf., par exemple, Jérôme Baschet, L'Étincelle zapatiste. Insurrection indienne et résistance planétaire, Paris, Denoël, 2002. Le sous-titre de cet ouvrage résume parfaitement cette ambiguité. 
d'une ancienne organisation dissoute, l'Armée insurgée mexicaine, n'avaient aucun lien avec le Chiapas et avec les peuples indigènes mais constituaient un authentique groupe révolutionnaire qui s'était fixé pour objectif de prendre le pouvoir par les armes. De même, au lendemain du soulèvement du $1^{\text {er }}$ janvier 1994, la rhétorique révolutionnaire a dominé les communiqués officiels de l'EzLn. Invoquant l'article 39 de la Constitution, qui reconnaît au peuple «le droit inaliénable de modifier la forme de son gouvernement», l'EZLN a déclaré «la guerre à l'armée fédérale mexicaine, principal pilier de la dictature exercée par le parti au pouvoir», et a appelé à l'instauration d'un «gouvernement révolutionnaire ${ }^{4}$. Cependant, au regard de la faiblesse des forces armées engagées, le but était avant tout de sortir de l'ombre et d'acquérir une nouvelle dimension sur la scène politique nationale, voire internationale.

Le ton révolutionnaire des premiers communiqués fit rapidement place à un discours plus modéré aux accents altermondialistes. Lorsque, le 13 janvier 2016, le sous-commandant Marcos ${ }^{5}$ déclara: «Peut-être la nouvelle morale politique doit-elle se construire en marge du pouvoir, comme un contrepoids pour exercer un contrôle sur celui-ci et l'obliger à obéir ${ }^{6}$ ", il n'appela pas au renversement du gouvernement mexicain, mais à la constitution d'un contre-pouvoir allant dans le sens des aspirations des indigènes chiapanèques, même s'il revendiqua la succession d'Emiliano Zapata, privant le gouvernement mexicain du monopole de la référence à ce mythe national ${ }^{7}$, et qu'il se déclara favorable à la révolution et à des changements politiques radicaux. C'est d'ailleurs le sens de la déclaration qu'il avait faite le 12 janvier 1994: "Nous ne pouvons pas et ne voulons pas imposer nos idées par la force des armes

4. Cf. Bertrand de La Grange et Maïté Rico, Sous-commandant Marcos. La géniale imposture, Paris, Plan-Ifrane, 1998, p. 22.

5. Rafael Sebastián Guillén Vicente, dit sous-commandant Marcos, né en 1957 à Tampico, est un militant altermondialiste mexicain. Influencé par la théologie de la libération pendant sa scolarité au collège jésuite puis à l'institut culturel de Tampico, puis par Louis Althusser, Michel Foucault et Karl Marx durant ses études de philosophie et de lettres à l'université nationale autonome de Mexico (UNAM), il fit quelques séjours au Chiapas, dans les années 1980, pour participer à des projets sociaux et syndicaux, tout en donnant des conférences sur l'administration des entreprises et des enseignements à l'Université autonome métropolitaine (UAM), à Mexico. Au sein de cette dernière, il aurait fréquenté des professeurs révolutionnaires, avant de démissionner en 1984 pour rejoindre l'EZLN, dont il est devenu le chef en 1986 et le porteparole en 1994. En mai 2014, il annonça qu'il quittait la direction de l'organisation révolutionnaire et qu'il prenait le nom de «sous-commandant Galeano », en hommage à un compagnon tué. En février 2016, le gouvernement mexicain abandonna les poursuites judiciaires engagées contre lui pour actes de rébellion et de terrorisme.

6. La Jornada Semanal, 14 janvier 1996.

7. Ibid. 
à la société civile mexicaine, à l'inverse du gouvernement, qui impose son projet national par la force des armes. Nous ne nous opposerons pas au processus électoral en $\operatorname{cours}^{8}$.» Reléguant au second plan l'action armée, il pouvait affirmer: "Nous sommes la seule guérilla qui accorde plus d'importance au discours qu'aux coups de feu.»

Le déséquilibre des forces armées engagées n'autorisait aucune surenchère rhétorique ni ne laissait espérer une victoire militaire, et la faible organisation politique des indigènes chiapanèques ne permettait aucune propagation de la rébellion. Mais les néozapatistes pouvaient envisager l'implantation durable au Chiapas d'un foyer de rébellion pouvant servir de modèle à d'autres peuples indigènes, et affaiblir, voire déstabiliser, le pouvoir fédéral en le forçant à mener une guerre de basse intensité, impopulaire au niveau national et international. C'est en ce sens que l'on peut considérer le mouvement néozapatiste comme un mouvement révolutionnaire dont l'objectif politique est moins indigéniste qu'antinéolibéral.

\section{Le néozapatisme est un mouvement antinéolibéral}

Le mouvement néozapatiste a rapidement levé le voile sur son projet réel, plus large que la défense de la cause indigène au Chiapas, lequel vise, au niveau du Mexique, à fédérer les mouvements indigènes pour faire pression sur le pouvoir et, au niveau mondial, à remettre en cause le néolibéralisme.

Sur le plan national, l'EZLN n'a jamais été une organisation purement chiapanèque, ni même indigéniste. On peut en ce sens évoquer la première déclaration de la forêt Lacandon - qui appelait à la destitution du président Salinas -, la Convention nationale démocratique - qui, en août 1994, a réuni six mille délégués d'organisations représentant tous les secteurs de la gauche mexicaine -, ou encore la quatrième déclaration de la forêt Lacandon - qui annonça la création du Front zapatiste de libération nationale, lequel intégra plusieurs secteurs de la gauche mexicaine dont les motivations ne se limitaient pas à la défense de la cause des indigènes chiapanèques.

De même, "L'Autre Campagne ", annoncée dans la sixième déclaration de la forêt Lacandon ${ }^{9}$ et créée en 2005 pour soutenir l'action des néozapatistes, visait la reconstruction de la nation mexicaine à partir de

8. Cité par Alma Guillermoprieto, La Guerre des ombres. Les racines de l'insurrection zapatiste an Chiapas, Paris, Dagorno, 1995, p. 52.

9. «Sixième déclaration de la forêt Lacandone», EnlaceZapatista.EZLN.org.mx, 30 juin 2005. 
la défense des plus humbles, sans faire clairement référence aux populations indigènes. Elle fut prolongée, durant l'été 2005, par sept rencontres au cours desquelles se constitua un réseau qui rassembla notamment des militants de partis antisystème, des syndicalistes et des membres d'organisations paysannes ou de groupes libertaires ou féministes. Enfin, en 2013 et 2014, l'EZLN fonda la "petite école zapatiste», qui devait permettre à des milliers de personnes de découvrir les réalisations concrètes de la gestion autonome du Chiapas lors de courts séjours dans les villages zapatistes. Ces initiatives avaient un point commun: l'expérimentation d'un nouveau modèle de société altermondialiste par le biais de la défense de la cause indigène.

En outre, les néozapatistes n'ont jamais souhaité enfermer leur action dans une dimension ethnique, et ont, au contraire, cherché à briser le mythe d'une société métissée qui aurait accordé aux indigènes leur juste place. Ils n’ont jamais opéré de séparation entre indigènes et nonindigènes. Ainsi, la marche de 2001, organisée en réaction au refus du gouvernement mexicain de réaliser une réforme constitutionnelle garantissant les droits indigènes, associait indigènes et non-indigènes, même si l'appellation qui lui a été donnée, la «Marche de ceux de la couleur de la terre », donnait une dimension ethnique, voire raciale, à l'événement.

Sur la scène internationale, l'action des néozapatistes s'est également caractérisée par la relégation au second plan de la cause indigène. Dès 1994, le néozapatisme a bénéficié d'une audience internationale qui lui était indispensable pour ne pas être étouffé et condamné au silence. Les comités de solidarité établis dans de nombreux pays lui ont assuré le soutien des opinions publiques. De même, de nombreux mouvements altermondialistes ont emprunté certains traits du néozapatisme, comme le mouvement des Indignés en Espagne, Occupy Wall Street aux États-Unis en septembre 2011, ou encore le mouvement français des «gilets jaunes» en 2018-201910. Mais c'est surtout la dimension anticapitaliste et altermondialiste du néozapatisme qui a inspiré ce soutien international.

Le 6 janvier 1996, le sous-commandant Marcos annonça, dans la déclaration de La Realidad pour l'humanité et contre le néolibéralisme ${ }^{11}$, l'organisation de la première Rencontre intercontinentale contre le néolibéralisme et pour l'humanité, qui s'est tenue dans la

10. Cf. «Le mouvement des gilets jaunes est-il zapatiste?» (entretien audio avec Jérôme Baschet), Reporterre.net, 10 avril 2019.

11. «Première déclaration de La Realidad contre le néolibéralisme et pour l'humanité», LaVoieDuJaguar.net, 6 janvier 1996. 
forêt Lacandon en août 1996 et à laquelle ont participé des délégations de mouvements communistes, des comités de soutien aux zapatistes, des mouvements autogestionnaires, paysans ou homosexuels provenant de quarante-deux pays, mais aussi des personnalités comme Eduardo Galeano, Alain Touraine, Danielle Mitterrand ou Gisèle Halimi, ainsi que des chefs guérilleros des années 1970, comme le Péruvien Hugo Blanco ou le Vénézuélien Douglas Bravo ${ }^{12}$. Au cours de cette rencontre, le sous-commandant Marcos a annoncé la mise en place d'un réseau de résistance intercontinental devant structurer la lutte contre le néolibéralisme. Cette rencontre internationale a apporté une forte impulsion aux mouvements altermondialistes, avant même les manifestations de Seattle en décembre 1999. Par la suite, six autres rencontres, ouvertement antinéolibérales, ont été organisées entre 1997 et 2015.

90 Dans le même sens, on peut citer la création, en décembre 2012, de La Sexta, un réseau mondial de luttes anticapitalistes formé par la fusion de L'Autre Campagne et de la Zezta Internazional, une structure rassemblant initialement les adhérents non mexicains de L'Autre Campagne.

Au regard des conditions de son émergence et de ses actions depuis 1994, le néozapatisme semble être essentiellement un mouvement anticapitaliste et altermondialiste qui a utilisé la défense de la cause indigène comme outil de mobilisation de la critique antinéolibérale autour d'un projet de gestion alternative. Le Chiapas est ainsi rapidement devenu un laboratoire où les idées néozapatistes ont fait l'objet de réalisations concrètes parvenant à améliorer les conditions de vie des indigènes chiapanèques.

\section{UNE DÉFENSE EFFECTIVE DE LA CAUSE INDIGÈNE}

Le néozapatisme a une dimension indigéniste et ne se résume pas à une simple instrumentalisation de la misère indigène à des fins politiques. Au-delà d'une critique du modèle néolibéral, auquel il reproche d'être responsable des inégalités socio-économiques et de la misère d'une partie de la population mexicaine, il a cherché à apporter une réponse à la colère des populations indigènes face à un gouvernement qui fait peu de cas de leur sort et a permis d'indéniables avancées en matière économique et sociale.

12. Cf. Chroniques intergalactiques EZLN. Première rencontre intercontinentale pour l'bumanité et contre le néolibéralisme, Paris, Aviva Presse, 1997. 


\section{Une récupération de la cause indigène}

Le développement du néozapatisme à partir du milieu des années 1990 a été favorisé par l'influence doctrinale de l'Église catholique et les effets pervers de la politique gouvernementale à l'égard des populations indigènes.

Dès les années 1960, l’Église catholique développa des programmes de soutien aux populations les plus défavorisées. Cette action, qui relevait à la fois de sa mission caritative et d'une volonté de freiner l'avancée du protestantisme, prit la forme d'une politique d' «inculturation de la foi » souhaitée par le concile Vatican II et d'un travail de terrain inspiré par la théologie de la libération ${ }^{13}$. Samuel Ruiz, évêque de San Cristóbal, bien connu pour son engagement en faveur de cette doctrine, joua un rôle essentiel, très influencé par la conférence épiscopale de Medellín de 1968, au cours de laquelle un message adressé par le pape Paul VI critiqua la violence institutionnalisée comme moyen de préserver la stabilité politique et rappela la mission de l'Église en faveur des pauvres ${ }^{14}$. En octobre 1974, pour commémorer la mémoire de Bartolomé de Las Casas, il organisa un congrès indigène qui réunit mille deux cent trente délégués du Chiapas; celui-ci rendit possible une meilleure structuration de la contestation sociale chiapanèque en suscitant la création de plusieurs organisations indigènes, notamment l'Association rurale des intérêts collectifs-Union des syndicats, dont les membres les plus influents rejoignirent l'EZLN ${ }^{15}$. Ce congrès suivait de deux ans le décret de Luis Echeverría qui avait accordé aux Lacandon plus de six cent mille hectares de terres, ce qui impliquait l'expulsion des colons Tzeltales et Choles installés dans la forêt Lacandon depuis la fin des années 1940, provoquant de nombreux affrontements entre paysans indigènes et métis, la répression contre les organisations indigènes et la multiplication des expulsions et des assassinats ${ }^{16}$.

En effet, les programmes de développement économique du Chiapas ont souvent été défavorables aux indigènes. Ainsi, dans les années 1940, une réforme agraire permit aux paysans de se voir distribuer des terres,

13. Cette démarche n'a pas été toujours bien perçue, notamment par une partie des Indiens attachés aux traditions et au catholicisme tridentin.

14. C'est d'ailleurs au cours de cette conférence que fut employée pour la première fois l'expression «théologie de la libération » par le père Gustavo Gutiérrez. Cf. Gustavo Gutiérrez, Théologie de la libération, Bruxelles, Lumen Vitae, 1974.

15. "¿Qué importancia tuvo el primer congreso indígena "Fray Bartolomé de Las Casas" ?", Nasdat.com.

16. Cf. Antonio Garcia de León, Chiapas: les comptes d'une rébellion, Paris, Syllepse, 1995. 
moins dans le but de lutter contre la misère que d'aider les riches propriétaires fonciers à développer l'élevage intensif, plus lucratif que l'exploitation d'haciendas par des paysans exploités. De même, la construction d'écoles et de dispensaires rapidement abandonnés relevait uniquement de la recherche par le Parti révolutionnaire institutionnel du soutien électoral des populations rurales.

Durant des décennies, le gouvernement mena une politique d'étouffement des populations indigènes au Chiapas qui passa par trois séries de mesures. Entre 1974 et 1984, il les concentra sur les hautes terres déjà très peuplées pour les isoler du reste de la population, constituant progressivement une vaste réserve indigène par le rachat des terres et des maisons des $\operatorname{Ladinos}^{17}$ redistribuées ensuite aux indigènes, et il les fixa dans la région en confiant à des membres du Parti révolutionnaire insti-

92 tutionnel le soin de protéger leur culture et leurs traditions. Il appliqua également une politique répressive, en renforçant la présence de la police et de l'armée aux limites des hautes terres, officiellement pour éviter une extension au Chiapas du conflit guatémaltèque par l'afflux de réfugiés, et en réformant le code pénal de 1990 pour redéfinir le délit d'atteinte à l'ordre public et aggraver les peines encourues en vue de prévenir toute rébellion. Enfin, il mit en œuvre une politique d'assistanat au détriment des activités productives, avec le programme de développement économique et social du Chiapas (1971-1976), le plan national des zones défavorisées et des groupes marginalisés (1977-1988) et le programme national de solidarité (1989).

Le gouvernement mexicain prit également une série de décisions qui, combinées, condamnèrent les indigènes chiapanèques à la précarité. En 1974, le président Echeverría signa un décret qui confiait à soixante-dix familles d'Indiens la gestion de la forêt Lacandon et interdisait aux colons tout nouveau défrichage pour gagner des terres sur la forêt. En novembre 1991, le président Salinas proposa au Congrès de réviser l'article 27 de la Constitution et de supprimer la «propriété sociale» octroyée aux communautés indigènes afin de permettre la création de fermes collectives, mettant fin à la redistribution de terres agricoles et permettant la vente des fermes communautaires, ce qui attira la convoitise de riches exploitants agricoles qui, depuis le décret de 1974, ne pouvaient plus s'étendre du fait de l'interdiction de défricher la forêt. Enfin, le gouvernement refusa d'accorder des subventions pour soutenir

17. En Amérique centrale, ce terme désigne les personnes dont la langue maternelle est l'espagnol et la culture hispanique, et éventuellement les métis et les Amérindiens assimilés. 
le prix du café, dont le cours mondial s'était effondré en 1989, causant la ruine des petits cultivateurs du Chiapas, qui avaient pourtant été encouragés à se lancer dans cette production grâce à des prêts consentis par les pouvoirs publics, ou les condamnant à vivre sous la menace d'une perte de leurs terres en cas de mauvaises récoltes et d'impossibilité de rembourser leurs emprunts.

Une telle politique très défavorable aux populations indigènes ne pouvait que susciter un sentiment de révolte. Le mouvement néozapatiste n'eut ainsi aucun mal à diffuser un discours anticapitaliste radical aux accents altermondialistes. Cependant, on peut mettre à son crédit un ensemble de réalisations qui ont profondément transformé les conditions de vie des indigènes chiapanèques.

\section{Une défense efficace de la cause indigène}

Les réalisations des néozapatistes au Chiapas sont essentiellement le fruit de la mise en ouvre des revendications politiques et administratives, économiques et sociales, et culturelles exposées dans la déclaration du $1^{\text {er }}$ mars 1994.

Sur le plan politique et administratif, les néozapatistes réclamaient l'organisation d'élections libres et la lutte contre la fraude électorale, la reconnaissance à l'EZLN du statut de force militaire permettant l'application du droit international, la libre administration du Chiapas, le droit à l'information, la destitution du gouverneur du Chiapas, l'abandon des poursuites contre les membres de l'EZLN, l'indépendance des commissions de défense des droits de l'homme, la création d'une Commission nationale pour la paix dans la justice et la dignité chargée de l'application des accords conclus entre l'EZLN et le gouvernement fédéral, la libération des prisonniers politiques et la fin des actions de l'armée et de la police.

Sur le plan économique et social, les néozapatistes demandaient l'accès de tous à l'électricité et la participation aux bénéfices de l'exploitation du pétrole, la révision de l'ALENA, une réforme agraire, la construction d'hôpitaux, l'installation de nouveaux médecins et un meilleur accès aux soins, la construction de logements et le développement d'infrastructures sportives et culturelles, la fin des discriminations, la reconnaissance du droit au travail et à un salaire minimum, l'annulation des dettes, la lutte contre la malnutrition, l'indemnisation des familles ayant subi des bombardements, l'acheminement de l'aide humanitaire aux victimes du conflit par des représentants des communautés autochtones et le respect des droits des femmes. 
Enfin, les revendications culturelles comportaient la lutte contre l'analphabétisme, l'accès à l'éducation gratuite, la reconnaissance et l'enseignement des langues indigènes et le respect de la dignité des peuples et cultures et traditions autochtones.

Les néozapatistes ne réclamaient pas l'indépendance du Chiapas, les indigènes se sentant mexicains, mais seulement l'autonomie afin de s'affranchir d'institutions jugées incompétentes, malhonnêtes et indifférentes aux intérêts des indigènes. C'est dans cette logique qu'ils ont mis en place un système administratif parallèle aux institutions officielles.

En décembre 1994 ont été créées trente-huit municipalités autonomes, puis, en 1995, des régions autonomes, les Aguascalientes. Le 16 février 1996, les accords de San Andrés, signés par l'EZLN et le gouvernement mexicain, ont proclamé le droit à l'autonomie des zones constituant

94 l'«habitat traditionnel» des indigènes, sous la forme de communautés (villages ou hameaux) ou de municipalités (communes) bénéficiant d'un droit d'association, ce qui conduisit à l'émergence d'une autonomie régionale confirmée les 8,9 et 10 août 2003 à Oventik avec la création des «caracoles». Ces accords prévoyaient également la mise en œuvre de programmes alternatifs en matière juridictionnelle, éducative et sanitaire, ce à quoi l'EzLN a ajouté des programmes de production agroécologique et de commercialisation alternative par des coopératives. Cinq "conseils de bon gouvernement», composés de délégués des municipalités autonomes zapatistes, ont été établis pour coordonner l'action des différentes communautés et répartir l'aide étrangère, corriger les déséquilibres de développement des municipalités, servir de médiateurs dans les conflits entre municipalités autonomes et entre celles-ci et les municipalités gouvernementales, mettre en place des projets productifs et représenter les communautés autonomes au niveau national et international; ils sont placés sous le contrôle du Comité indigène révolutionnaire clandestin-Commandement général de l'EZLN, officiellement pour prévenir les risques de corruption, d'arbitraire et d'injustice.

Des résultats concrets ont rapidement été obtenus, dans les domaines de la santé publique (développement de la vaccination et de la médecine préventive, création de cinq cents maisons de santé communautaires, de trois hôpitaux et de douze cliniques communautaires ou municipales, et formation de plus d'un millier de professionnels de la santé), de l'éducation (création de deux cents écoles primaires et de huit établissements d'enseignement secondaire), de l'économie (création de trois entrepôts d'approvisionnement qui desservent des centaines de magasins communautaires, et de coopératives autonomes de caféiculture et d'artisanat) et 
de la culture (création d'une maison d'édition et d'une station de radio). En revanche, les zapatistes ont souvent critiqué les coutumes ancestrales au nom des droits des femmes, des droits de l'homme et de la démocratie, et ont appelé les indigènes à accepter une évolution de leur culture.

L'apport des néozapatistes aux populations indigènes est indéniable. Même si la pauvreté n'a pas été éradiquée, les progrès sont sensibles et les néozapatistes y voient la preuve de l'efficacité de la politique alternative au libéralisme qu'ils prônent. Pour autant, les néozapatistes ne parviennent pas à tirer un profit politique, au niveau national, de leurs réalisations, et cela pour trois raisons.

D'abord, les indigènes adoptent un comportement opportuniste et accordent généralement leur soutien dans l'espoir que leurs conditions de vie s'en trouvent améliorées. Or l'administration officielle tend à privilégier les non-indigènes en échange de leur soutien au parti politique au pouvoir. De peur de voir les indigènes quitter les collectivités autonomes, l'EZLN leur demande donc de refuser les aides gouvernementales, mais elle se prive ainsi d'un soutien populaire fiable.

Ensuite, les néozapatistes se heurtent à l'indifférence de l'opinion publique mexicaine, les intérêts défendus pouvant être perçus comme très spécifiques à une partie de la population mexicaine qui a toujours fait l'objet de racisme: autant les indigènes du Chiapas se sentent mexicains, autant le reste de la population mexicaine se désintéresse de leur sort, voire les considère avec mépris.

Enfin, les néozapatistes entretiennent des rapports ambigus avec les partis politiques. Alors qu'ils se sont toujours opposés au Parti révolutionnaire institutionnel, ils ont soutenu une organisation politique issue d'une scission de ce dernier, le Parti de la révolution démocratique. Dans un second temps, ils ont refusé de soutenir ses candidats aux élections pour garder leur indépendance vis-à-vis des partis traditionnels. Enfin, ils ont fini par adopter un ton critique plus radical, en particulier au cours de la campagne électorale pour l'élection présidentielle de 2006 et ils ont condamné le Parti de la révolution démocratique pour la corruption de certains de ses membres et les liens qu'il entretenait avec des acteurs économiques. De même, les néozapatistes n’ont jamais toléré le racisme anti-indigène dont fait preuve une partie de la classe politique mexicaine, d'où un ton souvent très critique à l'égard de celle-ci.

Paradoxalement, cette marginalisation relative du néozapatisme au Mexique pourrait profiter aux indigènes du Chiapas. Le modèle altermondialiste expérimenté au niveau local ayant permis une amélioration de leurs conditions de vie, le gouvernement n'aurait intérêt à 
l'entraver que s'il risquait de se propager dans d'autres régions du pays et, à terme, de déstabiliser le système politique mexicain, ou de gêner la mise en œuvre de certains projets du gouvernement - par exemple, en matière de développement économique ou d'exploitation des ressources naturelles. Or la politique d'endiguement menée à travers la conduite d'une guerre de basse intensité permet au gouvernement d'éviter que les pratiques d'administration autonome inspirées par les néozapatistes ne prennent trop d'ampleur. C'est là le prix à payer pour que la cause indigène bénéficie de la poursuite de l'expérience zapatiste.

Même s'il est d'usage de l'associer à la cause indigène, le néozapatisme ne se résume pas à la défense de celle-ci. Mouvement révolutionnaire à l'origine, il a certes épousé le combat pour le respect des droits des peuples indigènes $d u$ Chiapas, mais son objectif principal est devenu la lutte contre le néolibéralisme dans la logique de la mouvance altermondialiste, ce dont ont profité les indigènes, qui ont vu leurs conditions de vie sensiblement améliorées par la mise en place de structures de gestion alternatives dans certaines régions du Chiapas. 